\title{
Measurement of the V2I Massive Radio Channel with the MaMIMOSA Sounder in a Suburban Environment
}

\author{
D.P. Gaillot* ${ }^{* \dagger}$, P. Laly*, N. Dahmouni*, G. Delbarre*, M. Van den Bossche ${ }^{\ddagger}$, G. Vermeeren ${ }^{\ddagger}$, E. Tanghe ${ }^{\ddagger}$, \\ E.P. Simon* ${ }^{* \dagger}$, W. Joseph ${ }^{\ddagger}$, L. Martens ${ }^{\ddagger}$, M. Liénard* \\ *IEMN (UMR 8520), University of Lille, Villeneuve d'Ascq, France. martine.liénard@univ-lille.fr \\ †IRCICA (USR 3380), University of Lille, Villeneuve d'Ascq, France. davy.gaillot@univ-lille.fr \\ ‡INTEC - WAVES, Ghent University, Ghent, Belgium. wout.joseph@ugent.be
}

\begin{abstract}
This paper presents the first V2I channel sounding campaign with the real-time MaMIMOSA massive radio channel sounder. This equipment has been jointly developed by ULille (FR) and UGhent (BE) for 5G V2X applications. The system is equipped with a massive 64-antenna array for $T x$ whereas up to 8 individual antennas can be deployed for Rx. The MaMIMOSA hardware and software capabilities allow to freely adapting the sounding parameters to the investigated scenario demonstrating its versatility and flexibility. Radio channels were measured at $5.89 \mathrm{GHz}$ with $80 \mathrm{MHz}$ bandwidth at the ULille campus using with a vehicle speed up to $60 \mathrm{~km} / \mathrm{h}$. In addition to this suburban mobility scenario, Obstructed Line-Of-Sight static radio channels were collected for a roadside to parking setup to study the influence of vegetation. Very preliminary Doppler characteristics are reported from the measured radio channels. A deeper analysis will be included in the final paper.
\end{abstract}

Index Terms-Massive MIMO channel sounding, V2X, propagation, measurements.

\section{INTRODUCTION}

In our modern world where mobile connectivity has become a necessity for many network users, there is a need for technological advances and computer abilities to provide faster, smarter and safer wireless networks [1]. The upcoming 5G NR (New Radio) being currently defined by 3GPP [2], [3] is expected to reach larger data rates with improved networks efficiency compared to previous legacy standards such as $4 \mathrm{G}$ LTE. Moreover, it widens its original radio mobile domain of application by including machines in industrial setups, vehicular communications (vehicle-to-everything or V2X) [4] and smart cities [5], [6]. These use cases encompass three fundamentally different dimensions: enhanced mobile broadband (eMBB), massive machine type communications (mMTC) and ultra-reliable low latency communications (URLLC). Among novel technologies expected to deliver the promised specifications of 5G NR, Massive MIMO [7] and the use of larger bandwidths at millimeter wave $(\mathrm{mmW})$ frequencies are the most widely investigated in the research community. Massive MIMO is an asymptotic extension of multi-user MIMO (MUMIMO) where the number of transmitting antennas is very large compared to previous MIMO techniqies. This enables simultaneously serving many user equipments (UE) within the same frequency-time slot even if they generally are equipped with one antennas one antenna [8], [9]. Early works such as [10], [11] present the fundamental information, theoretical aspects as well as limits of the massive MIMO technology.

Beyond the fact that the main benefits envisioned by massive MIMO must be evaluated (e.g. reduction in latency on the air interface, multiple-layer access simplification or even robustness to undesired or intentional jamming [12]), legacy propagation models used in network planning tools must be revisited accordingly. Furthermore, the wide-sense stationarity (WSS) uncorrelated scattering (US) assumption WSSUS [?] of the channel are not often verified in realistic dynamic environments from the Tx array side. The violation of the WSSUS assumptions which can happen in practice in vehicular scenarios [13] avoid a simplified statistical description of channels. It follows real-time massive MIMO systems must be used to assess the WSSUS assumtions and is a requisite to grasp the time-varying spatial characteristics of the radio channel. This approach can be rather cost-expensive due to the architecture complexity [14] resulting in RF impairments or synchronization issues between the massive array and the UEs.

To this end, a real-time 64 x 16 massive MIMO radio channel sounder operating between 2 and $12 \mathrm{GHz}$ with $80 \mathrm{MHz}$ bandwidth has been jointly developed by the University of Lille (France) and Ghent (Belgium) for 5G mobility scenarios such as V2X communications [15]. The architecture relies on the physical and reconfigurable software radio channel sounder MIMOSA [16]. MaMIMOSA has been designed to fulfill all the constraints identified in time-varying massive MIMO channels such as high Doppler resolution with a large Doppler span, spatial antenna selection, compactness, energy consumption, etc. The sounding parameters of the developed massive system can be freely selected depending on the studied environment resulting in the measured massive MIMO radio channel without the need for additional tedious post-processing. The MaMIMOSA antenna array is a $10 \times 10$ vertical planar antenna array built by the University of Ghent. 64 elements of the array are SMA-connected to the Tx unit using coaxial cables whereas the 36 remaining antennas at the 
perimeter are used as dummies to attenuate the finite array side effects.

In this work, MaMIMOSA was setup to perform up to 64 x 8 massive radio channel measurements at $5.89 \mathrm{GHz}$ with $80 \mathrm{MHz}$ bandwidth for V2I communications. In a first set of measurements, $64 \times 1$ time-varying radio channels were assessed on the scientific campus of the University of Lille with a vehicle speed up to $60 \mathrm{~km} / \mathrm{h}$. In a second set, static Obstructed Line-Of-Sight (OLOS) 64 x 8 radio channels were measured between the transmitter located on the roadside and receiver on an adjacent parking lot to study the influence of vegetation. Since the campaign is very recent, only an early analysis of the Doppler characteristics were evaluated from the measured channels. A deeper analysis of the fading and Doppler characteristics will be presented in the final paper. Nonetheless, The campaign already highlights the potential of MaMIMOSA to faithfully grasp the main characteristics of massive radio channels which can be subsequently used for developing realistic radio channel models.

\section{MAMIMOSA FRAME STRUCTURE}

\section{A. Streaming mode for time-varying scenarios}

When a scenario with large mobility is considered like for $\mathrm{v} 2 \mathrm{X}$ communications, the MaMIMOSA streaming mode is selected and is associated the frame structure illustrated in Fig. 1(a). Each frame consists in one $51.2 \mu$ s preamble subsequently followed by 128 blocks of $8121.92 \mu$ s OFDM symbols including the cyclic prefix. The total frame duration to measure a single $64 \times 16$ massive MIMO matrix is $\sim 1 \mathrm{~ms}$ and $\sim 125 \mathrm{~ms}$. Hence, the maximum Doppler span is \pm 512 $\mathrm{Hz}(93 \mathrm{~km} / \mathrm{h}$ maximum speed) with $8 \mathrm{~Hz}$ Doppler resolution. The preamble provides the time synchronization of the frame required to decode the OFDM symbols. Each OFDM symbol corresponds to an 8-antenna subarray out of 64 . The time duration between consecutive frames was manually set to 500 $\mathrm{ms}$. The total number of transmitted OFDM subcarriers per symbol $N_{t}$ is 8192 which can be uniformly distributed on each antenna element using interleaved OFDM. For instance, the frequency space $\delta F$ between the subcarriers of a single antenna is $97.66 \mathrm{kHz}$, meanwhile the frequency space of between subcarriers is $12.21 \mathrm{kHz}$. The automatic gain control (ACG) is performed with the cyclic prefix for each symbol and is used to correct the measured MIMO matrix. The reader is encouraged to read the following references to learn more about MaMIMOSA's architecture and signal processing [15], [16]. The non-stationary fading process of vehicular channel can be characterized by assuming local stationarity for a finite region in time and frequency. We reported in a recent work a stationarity time of $567 \mathrm{~ms}$ for the same scenario at 1.35 $\mathrm{GHz}$ [13] and similar speeds. Hence, it is assumed that the measurement time used to obtain the estimates of the Doppler and delay spreads are within the stationarity region.

\section{B. Streamshot mode for static scenarios}

When the time-variation of the radio channel is not the purpose of the measurement or simply because the radio channel is supposed to be static in nature, the streamshot mode of MaMIMOSA can be selected and is associated the frame structure illustrated in Fig. 1(b). Each frame consists in one $51.2 \mu$ s preamble subsequently followed by a single block of $8121.92 \mu \mathrm{s}$ OFDM symbols including the cyclic prefix. The total frame duration to measure a single $64 \times 16$ massive MIMO matrix is $\sim 1 \mathrm{~ms}$. The time duration between consecutive frames was also manually set to $500 \mathrm{~ms}$. Since time-synchronization is performed to measure a single massive radio channel, the Doppler information is lost. However, this mode relaxes the streaming and processing constraints within the FPGA to the hard drive in contrast to the streaming mode.

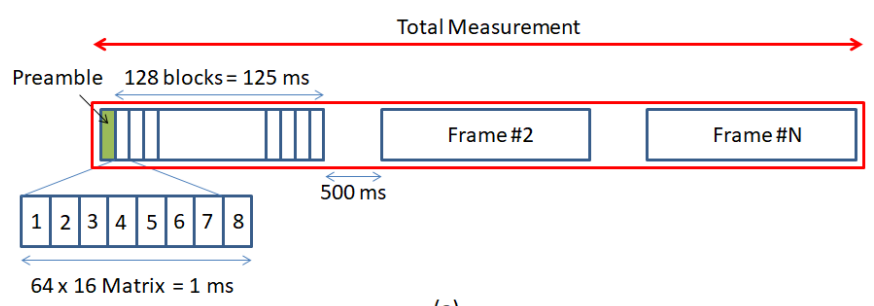

(a)

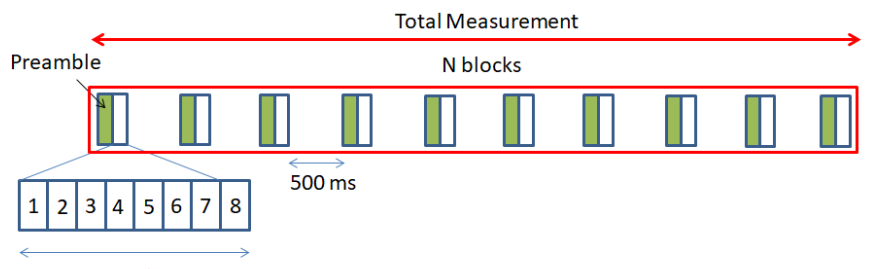

$64 \times 16$ Matrix $=1 \mathrm{~ms}$

(b)

Fig. 1. MaMIMOSA frame structure for (a) textitstremaing and (b) streamshot mode.

\section{MeAsurement CAMPAign DESCRiPtion}

The massive 64-antenna array was placed on a tripod at same location on the sidewalk during the whole measurement campaign. The emitted power per RF chain was set to $0 \mathrm{dBm}$ during the whole measurement campaign knowing that the maximum power per RF chain is $30 \mathrm{dBm}$.

\section{A. V2I scenarios}

Figure. 2 depicts a schematic top view of the V2I measurement campaign at the ULille campus. Tx was placed such that its main radiating lobe was parallel to the main boulevard. The single receiver antenna $\mathrm{Rx}$ was placed on a van rooftop and is the same as the elementary antenna of the array. The van performed a drive test along the boulevard with maximum speed of $60 \mathrm{~km} / \mathrm{h}$ toward Tx. The massive radio channel was measured during 118 frames with the streaming mode for a total recording time of $\sim 54$ seconds. Figure. 3 presents a picture from the rear side of the $\mathrm{Tx}$ array with the van completing its drive test.

\section{B. Static scenarios}

In addition, Figure. 4 depicts a picture of the 4 OLOS measurement scenarios between the Tx array (located at same 


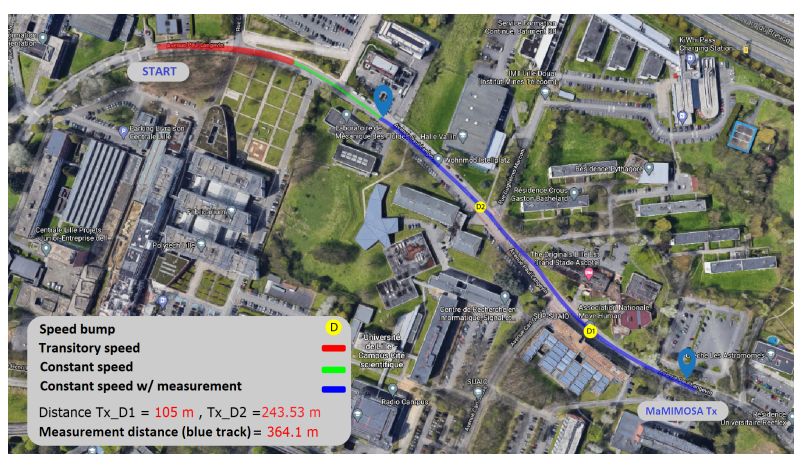

Fig. 2. schematic top view of the V2I measurement campaign at the ULille campus.

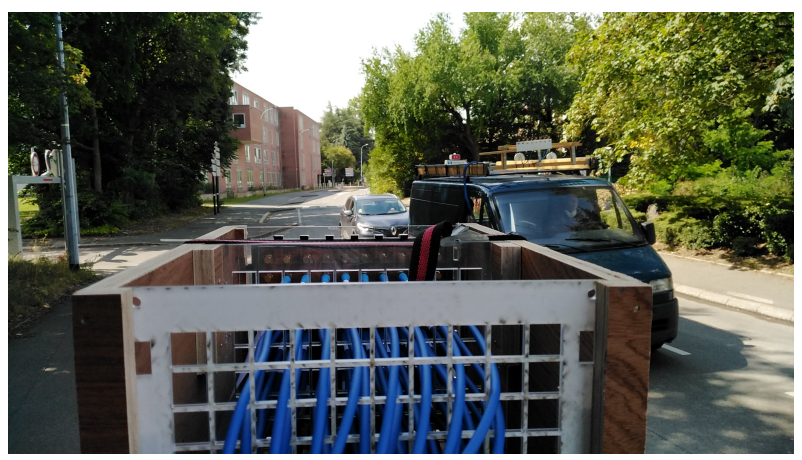

Fig. 3. Picture from the rear side of the Tx array with the van completing its drive test. The Rx antenna is one of the 8 antennas located on the van rooftop.

place than for the V2I scenario) and 8-antenna Rx array. Tx array was facing the building. The measurement recently took place at the end of the summer such that the foliage of the trees separating the sidewalk and parking was dense. The massive radio channel was measured during 236 frames with the streamshot mode.

\section{PReliminary Doppler Results}

Figure. 5 presents the Doppler shift measured for the V2I scenario averaged for Tx antenna 1 as a function of the number of frames ( $\mathrm{Tx}$ - Rx distance). The associated vehicle speed computed as the maximum in the Doppler spectrum for each frame is in good agreement with the speed maintained during the measurement. A spike can be observed and is associated

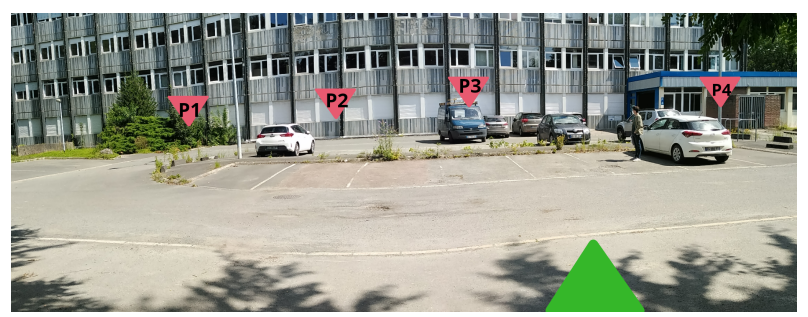

Fig. 4. Schematic top view of the 4 OLOS static measurement positions. The green triangle corresponds to the Tx position on the sidewalk behind the trees observable by their shadows on the ground.
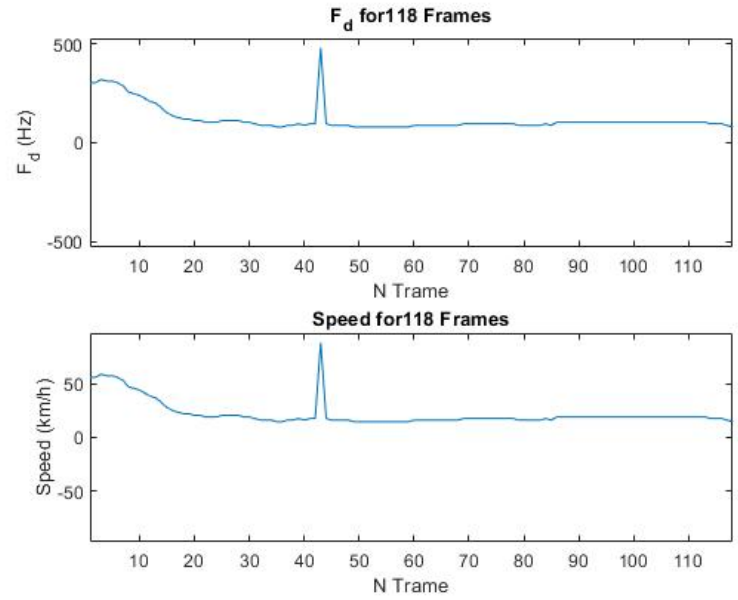

Fig. 5. Doppler shift (in $\mathrm{Hz}$ ) and equivalent speed (in $\mathrm{km} / \mathrm{h}$ ) as a function of the number of frames (i.e. Tx - Rx distance).

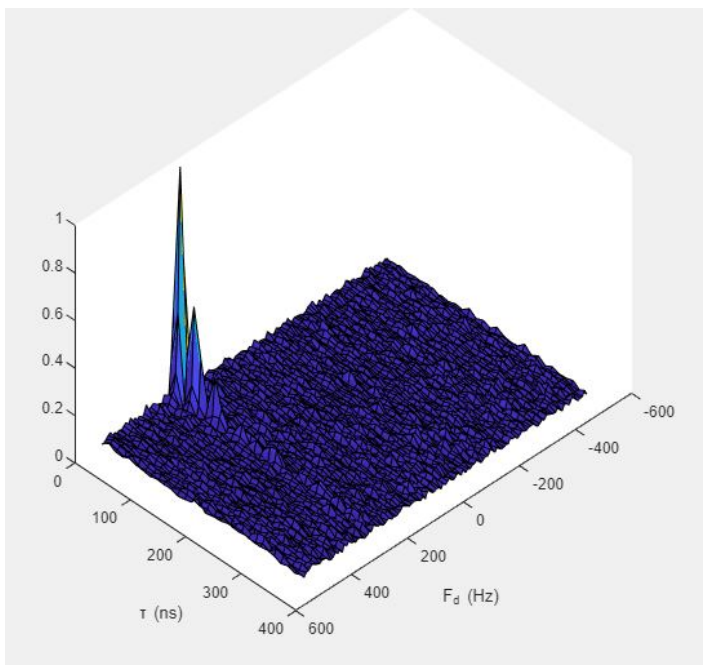

Fig. 6. Example of estimated Delay-Doppler spectrum for frame \#10 and Tx antenna 1

with the first speed bump labeled D2 along the main boulevard as seen in Fig. 2.

In addition, Fig. 6 presents the estimated Delay-Doppler profile as a function of Tx - Rx distance for frame \#10 and Tx antenna 1 as an example. The spectrum indicates the presence of several multipath components with similar Doppler shift values as the principal Doppler shift due to the van.

\section{CONClusion}

In this contribution, the real-time $64 \times 16$ massive MIMO sounder called MaMIMOSA based on space-frequency division multiplexing and antenna subarray switching has been used for a measurement campaign at $5.89 \mathrm{GHz}$ with $80 \mathrm{MHz}$ bandwidth on the University of Lille Campus. using different Massive transmitter-receiver configurations with vehicle speed up to $60 \mathrm{~km} / \mathrm{h}$. In addition, the influence of vegetation was investigated thanks to the measurement of OLOS static 
radio channels from the roadside to a parking lot. The very preliminary Doppler characteristics were evaluated from the measured radio channels and are shown to agree well with the campaign setup. A deeper analysis if the radio channel fading and Doppler characteristics for both time-varying and OLOS scenarios will be presented in the final paper.

\section{ACKNOWLEDGMENT}

This work was supported through the OS4 SMARTIES research program by the ELSAT2020 project co-financed by the European Union with the European Regional Development Fund, the French state, and the Hauts-de-France Region Council.

\section{REFERENCES}

[1] I. F. Akyildiz, S. Nie, S.-C. Lin, and M. Chandrasekaran, "5g roadmap: 10 key enabling technologies," Computer Networks, vol. 106, pp. $17-48$, 2016. [Online]. Available: http://www.sciencedirect.com/science/article/pii/S1389128616301918

[2] S. Parkvall, E. Dahlman, A. Furuskar, and M. Frenne, "Nr: The new 5g radio access technology," IEEE Communications Standards Magazine, vol. 1, no. 4, pp. 24-30, Dec 2017.

[3] 3GPP, 3GPP Technical Report Technical Specification Group Radio Access Network; Study on NR Industrial Internet of Things (IoT), Release 16, 2015.

[4] D. Phan-Huy, M. Sternad, and T. Svensson, "Making 5g adaptive antennas work for very fast moving vehicles," IEEE Intelligent Transportation Systems Magazine, vol. 7, no. 2, pp. 71-84, Summer 2015.

[5] A. Osseiran, F. Boccardi, V. Braun, K. Kusume, P. Marsch, M. Maternia, O. Queseth, M. Schellmann, H. Schotten, H. Taoka, H. Tullberg, M. A. Uusitalo, B. Timus, and M. Fallgren, "Scenarios for $5 \mathrm{~g}$ mobile and wireless communications: the vision of the metis project," IEEE Communications Magazine, vol. 52, no. 5, pp. 26-35, May 2014.

[6] A. Gupta and R. K. Jha, "A survey of $5 \mathrm{~g}$ network: Architecture and emerging technologies," IEEE Access, vol. 3, pp. 1206-1232, 2015.

[7] T. L. Marzetta, "Noncooperative cellular wireless with unlimited numbers of base station antennas," IEEE Transactions on Wireless Communications, vol. 9, no. 11, pp. 3590-3600, November 2010.

[8] N. B. Labs., $5 G$ New Radio (NR) : Physical Layer Overview and Performance., 2018. [Online]. Available: http://ctw2018.ieeectw.org/files/2018/05/5G-NR-CTW-final.pdf

[9] K. Technologies., Field Testing in 5G NR., 2018. [Online]. Available: https://literature.cdn.keysight.com/litweb/pdf/5992-3299EN.pdf

[10] E. Björnson, J. Hoydis, M. Kountouris, and M. Debbah, "Massive mimo systems with non-ideal hardware: Energy efficiency, estimation, and capacity limits," IEEE Transactions on Information Theory, vol. 60, no. 11, pp. 7112-7139, Nov 2014.

[11] J. Jose, A. Ashikhmin, T. L. Marzetta, and S. Vishwanath, "Pilot contamination and precoding in multi-cell tdd systems," IEEE Transactions on Wireless Communications, vol. 10, no. 8, pp. 2640-2651, August 2011.

[12] E. G. Larsson, O. Edfors, F. Tufvesson, and T. L. Marzetta, "Massive mimo for next generation wireless systems," IEEE Communications Magazine, vol. 52, no. 2, pp. 186-195, February 2014.

[13] M. Yusuf, E. Tanghe, F. Challita, P. Laly, D. Gaillot, M. Lienard, L. Martens, and W. Joseph, "Stationarity analysis of v2i radio channel in a suburban environment," IEEE Transactions on Vehicular Technology, pp. 1-1, 2019.

[14] J. Vieira, S. Malkowsky, K. Nieman, Z. Miers, N. Kundargi, L. Liu, I. Wong, V. Öwall, O. Edfors, and F. Tufvesson, "A flexible 100-antenna testbed for massive mimo," in 2014 IEEE Globecom Workshops (GC Wkshps), Dec 2014, pp. 287-293.

[15] P. Laly, D. P. Gaillot, G. Delbarre, M. V. d. Bossche, G. Vermeeren, F. Challita, E. Tanghe, E. P. Simon, W. Joseph, L. Martens, and M. Liénard, "Massive radio channel sounder architecture for $5 \mathrm{~g}$ mobility scenarios: Mamimosa," in 2020 14th European Conference on Antennas and Propagation (EuCAP), 2020, pp. 1-5.
[16] P. Laly, D. P. Gaillot, M. Lienard, P. Degauque, E. Tanghe, W. Joseph, and L. Martens, "Flexible real-time mimo channel sounder for multidimensional polarimetric parameter estimation," in 2015 IEEE Conference on Antenna Measurements Applications (CAMA), Nov 2015, pp. 1-3. 